\title{
EXISTENCE OF WEAK SOLUTIONS TO STOCHASTIC DIFFERENTIAL EQUATIONS IN THE PLANE WITH CONTINUOUS COEFFICIENTS
}

\author{
BY \\ J. YEH ${ }^{1}$
}

ABSTRACT. Let $B$ be a 2-parameter Brownian motion on $\mathbf{R}_{+}^{2}$. Consider the nonMarkovian stochastic differential system in 2-parameter

$$
\begin{cases}d X(z)=\alpha(z, X) d B(z)+\beta(z, X) d z & \text { for } z \in \mathbf{R}_{+}^{2}, \\ X(z)=\xi & \text { for } z \in \partial \mathbf{R}_{+}^{2}\end{cases}
$$
i.e.,

$$
\left\{\begin{array}{l}
X(z)=X(0)+\int_{R_{z}} \alpha(\zeta, X) d B(\zeta)+\int_{R_{z}} \beta(\zeta, X) d \zeta \text { for } z \in \mathbf{R}_{+}^{2}, \\
X(0)=\xi
\end{array}\right.
$$

where $R_{z}=[0, s] \times[0, t]$ for $z=(s, t) \in \mathbf{R}_{+}^{2}$. An existence theorem for weak solutions of the system is proved in this paper. Under the assumption that $\alpha$ and $\beta$ satisfy a continuity condition and a growth condition and $\mathbf{E}\left[\xi^{6}\right]<\infty$, it is shown that there exist a 2-parameter stochastic process $X$ and a 2-parameter Brownian motion $B$ on some probability space satisfying the stochastic integral equation above, with $X(0)$ having the same probability distribution as $\xi$.

0. Introduction. Let a partial ordering be introduced in $\mathbf{R}_{+}^{2}$ by defining $z \leqslant z^{\prime}$ when $s \leqslant s^{\prime}$ and $t \leqslant t^{\prime}$ for $z=(s, t)$ and $z^{\prime}=\left(s^{\prime}, t^{\prime}\right)$ in $\mathbf{R}_{+}^{2}$. We write $z<z^{\prime}$ when $s<s^{\prime}$ and $t<t^{\prime}$. Similarly we write $z \wedge z^{\prime}$ for $\left(s \wedge s^{\prime}, t \wedge t^{\prime}\right)$ and $z \vee z^{\prime}$ for $\left(s \vee s^{\prime}, t \vee t^{\prime}\right)$. We use the notations $R_{z}$ and $R_{s, t}$ for $[0, s] \times[0, t]$ and the notation $\left(z, z^{\prime}\right]$ for $\left(s, s^{\prime}\right] \times\left(t, t^{\prime}\right]$ when $z<z^{\prime}$. Likewise we write $\left[z, z^{\prime}\right)$ for $\left[s, s^{\prime}\right) \times\left[t, t^{\prime}\right)$ and $\left[z, z^{\prime}\right]$ for $\left[s, s^{\prime}\right] \times\left[t, t^{\prime}\right]$.

Throughout this article, by a 2-parameter stochastic process on a probability space $(\Omega, \mathfrak{F}, P)$ we mean a real valued function $X$ on $\mathbf{R}_{+}^{2} \times \Omega$ such that $X(z, \cdot)$ is $\mathfrak{F} / \mathfrak{B}(\mathbf{R})$ measurable for every $z \in \mathbf{R}_{+}^{2}$. For such a process $X$ we write $X_{z}, X_{s, t}, X(z)$ and $X(s, t)$ for $X(z, \cdot)$ when $z=(s, t) \in \mathbf{R}_{+}^{2}$. For $z<z^{\prime}$ we use the notation $X\left(\left(z, z^{\prime}\right]\right)$ for $X\left(s^{\prime}, t^{\prime}\right)-X\left(s, t^{\prime}\right)-X\left(s^{\prime}, t\right)+X(s, t)$. We write $\partial X=0$ when $X_{z}=0$ for $z$ in the boundary $\partial \mathbf{R}_{+}^{2}$ of $\mathbf{R}_{+}^{2}$.

Received by the editors April 12, 1984 and, in revised form, September 12, 1984.

1980 Mathematics Subject Classification. Primary 60H10,60H05, 60H20.

Key words and phrases. Brownian sheet, stochastic differential equations, tightness, Kolmogorov condition.

${ }^{1}$ The author wishes to express his appreciation to the referee for the improvements made on the original version of this paper. 
Let $\left(\Omega, \mathfrak{F}, P ; \mathfrak{F}_{z}, z \in \mathbf{R}_{+}^{2}\right)$, or briefly $\left(\Omega, \mathfrak{F}, P ; \mathfrak{F}_{z}\right)$, be a probability space with a family $\left\{\mathfrak{F}_{z}, z \in \mathbf{R}_{+}^{2}\right\}$ of sub- $\sigma$-fields of $\mathfrak{F}$ satisfying the usual conditions. Consider the stochastic differential equation of non-Markovian type in the plane

$$
d X_{z}=\alpha(z, X) d B_{z}+\beta(z, X) d z,
$$

i.e.,

$$
X_{s, t}-X_{0, t}-X_{s, 0}+X_{0,0}=\int_{R_{z}} \alpha(\zeta, X) d B_{\zeta}+\int_{R_{z}} \beta(\zeta, X) d \zeta
$$

for $z=(s, t) \in \mathbf{R}_{+}^{2}$, where $B$ is an $\left\{\mathfrak{F}_{z}\right\}$-Brownian motion on $\left(\Omega, \mathfrak{F}, P ; \mathfrak{F}_{z}\right)$ with $\partial B=0$ and $d z$ denotes integration with respect to the Lebesgue measure on $\mathbf{R}_{+}^{2}$. The coefficients $\alpha$ and $\beta$ are real valued functions on $\mathbf{R}_{+}^{2} \times W$, where $W$ is the space of all continuous real valued functions on $\mathbf{R}_{+}^{2}$, satisfying certain measurability conditions which imply that for each $\omega \in \Omega, \alpha(z, X(\cdot, \omega))$ and $\beta(z, X(\cdot, \omega))$ depend only on that part of the sample function $X(\cdot, \omega)$ of $X$ which precedes $z$ in the sense of the partial ordering of $\mathbf{R}_{+}^{2}$.

In [18] we showed that under a Lipschitz condition on $\alpha$ and $\beta$ the solution of the stochastic differential equation, if it exists, is pathwise unique. We showed also that if $\alpha$ and $\beta$ satisfy, in addition to the Lipschitz condition, a certain order of growth condition (see condition (C2) in $\$ 1$ ), then for every $\left\{\mathfrak{F}_{z}\right\}$-adapted 2-parameter stochastic process $Z$ defined on $\partial \mathbf{R}_{+}^{2}$ and having a locally bounded second moment on $\partial \mathbf{R}_{+}^{2}$ the stochastic differential equation has a strong solution whose restriction to $\partial \mathbf{R}_{+}^{2}$ is equal to $Z$.

Let us now replace the Lipschitz condition in [18] by a continuity condition (see condition (C1) in $\S 1$ ) on $\alpha$ and $\beta$. Under the assumption of $(\mathrm{C} 1)$ and $(\mathrm{C} 2)$ on the coefficients $\alpha$ and $\beta$ and with an $\mathfrak{F}_{0}$-measurable random variable $\xi$ on $(\Omega, \mathfrak{F}, P$ ), we consider the stochastic differential equation with the boundary condition $X_{z}=\xi$ for $z \in \partial \mathbf{R}_{+}^{2}$, i.e., we consider the stochastic differential system

$$
\begin{gathered}
X(z)=X(0)+\int_{R_{z}} \alpha(\zeta, X) d B_{\zeta}+\int_{R_{z}} \beta(\zeta, X) d \zeta \quad \text { for } z \in \mathbf{R}_{+}^{2}, \\
X(0)=\xi .
\end{gathered}
$$

The main result of this paper is that when $\mathbf{E}\left[\xi^{6}\right]<\infty$, the stochastic differential system has a weak solution.

A particular case of the stochastic differential equation (0.1), namely the Markovian type,

$$
X(z)=X(0)+\int_{R_{z}} a(X(\zeta)) d B_{\zeta}+\int_{R_{z}} b(X(\zeta)) d \zeta \quad \text { for } z \in \mathbf{R}_{+}^{2},
$$

where the coefficients $a$ and $b$ are real valued functions on $\mathbf{R}$, has been treated by $C$. Tudor [14]. He showed the existence of a weak solution of (0.3) under the assumption that $a$ is continuous and satisfies the boundedness condition $0<C_{1} \leqslant a^{2}(x) \leqslant$ $C_{2}$ for $x \in \mathbf{R}$ and $b$ is measurable and bounded.

In treating (0.1), we adopt the approach in Watanabe [16] for stochastic differential equations in one-parameter with continuous coefficients. The contents of the 
present paper are as follows. In $\$ 1$ we give the definitions that are needed in the paper. In Theorem 2.2 in $\$ 2$ we show that the stochastic differential system $(0.1)$ and (0.2) has a solution when the coefficients $\alpha$ and $\beta$ in $(0.1)$ are step functions in $z \in \mathbf{R}_{+}^{2}$ satisfying the order of growth condition (C2) in $\S 1$ and the random variable $\xi$ in $(0.2)$ has $\mathbf{E}\left[\xi^{2}\right]<\infty$. When $\alpha$ and $\beta$ are continuous on $\mathbf{R}_{+}^{2} \times W$ they can be approximated by sequences of step functions $\alpha_{l}$ and $\beta_{l}, l=1,2, \ldots$, which inherit the order of growth condition from $\alpha$ and $\beta$. The stochastic differential system (0.1) and (0.2), with $\alpha_{l}$ and $\beta_{l}$ as coefficients, then has a solution $X_{l}$ for $l=1,2, \ldots$

Proposition 3.1 in $\S 3$ states that the moments of $X_{l}, l=1,2, \ldots$, are uniformly bounded. Specifically, for every $n=2$ or $n \geqslant 4$ and $T>0$ there exists a constant $C_{n, T} \geqslant 1$ such that

$$
\mathbf{E}\left[\left|X_{l}(z)\right|^{n}\right] \leqslant\left\{1+\mathbf{E}\left[|\xi|^{n}\right]\right\} e^{2 C_{n, T^{s t}}}-1
$$

for $z=(s, t) \leqslant(T, T)$ and $l=1,2, \ldots$ The estimate $(0.4)$ is an extension of the estimate due to J. Reid [11] for a single solution. By means of (0.4) we show in Proposition 3.2 that if $\mathbf{E}\left[\xi^{6}\right]<\infty$, then $X_{l}, l=1,2, \ldots$, satisfy a uniform Kolmogorov condition in the sense that for every $T>0$ there exists $\Gamma_{T}>0$ such that

$$
\mathbf{E}\left[\left|X_{l}(z)-X_{l}\left(z^{\prime}\right)\right|^{6}\right] \leqslant \Gamma_{T} d\left(z, z^{\prime}\right)^{3}
$$

for all $z, z^{\prime} \leqslant(T, T)$ and $l=1,2, \ldots$, where $d$ is the Euclidean metric on $\mathbf{R}_{+}^{2}$.

The condition (0.5) implies that for some subsequence $l(k), k=1,2, \ldots$, of $l=1,2, \ldots$, there exist continuous 2-parameter stochastic processes $X_{k}^{*}, k=1,2, \ldots$, and $X^{*}$ on some probability space satisfying the conditions that $X_{k}^{*}$ and $X_{l(k)}$ have the same probability distribution and that $X_{k}^{*}$ converges a.s. to $X^{*}$ in the topology on $W$ of uniform convergence on every bounded subset of $\mathbf{R}_{+}^{2}$. Let $\left\{\mathfrak{F}_{\infty, z}, z \in \mathbf{R}_{+}^{2}\right\}$ be the standard filtration associated with $X^{*}$. In Theorem 4.1 in $\$ 4$ we show that under the conditions (C1) and (C2) on $\alpha$ and $\beta$ the 2-parameter stochastic process $M^{*}$ defined by

$$
M^{*}(z)=X^{*}(z)-X^{*}(0)-\int_{R_{z}} \beta\left(\zeta, X^{*}\right) d \zeta \quad \text { for } z \in \mathbf{R}_{+}^{2}
$$

is a continuous, square integrable, $\left\{\mathfrak{F}_{\infty, z}\right\}$-strong martingale and furthermore a 2-parameter increasing process $\left\langle M^{*}\right\rangle$ associated with $M^{*}$ is given by

$$
\left\langle M^{*}\right\rangle(z)=\int_{R_{z}} \alpha\left(\zeta, X^{*}\right)^{2} d \zeta \quad \text { for } z \in \mathbf{R}_{+}^{2}
$$

so that there exists an extension of the probability space on which $X^{*}$ is based, with a 2-parameter Brownian motion $B^{*}$ on it satisfying

$$
X^{*}(z)=X^{*}(0)+\int_{R_{z}} \alpha\left(\zeta, X^{*}\right) d B_{\zeta}^{*}+\int_{R_{z}} \beta\left(\zeta, X^{*}\right) d \zeta \quad \text { for } z \in \mathbf{R}_{+}^{2}
$$

with $X^{*}(0)$ having the same probability distribution as $\xi$.

Equations in this paper, except for those in a proof, will be numbered as $(m, n)$ with $m$ indicating the section in which the equation appears. Equations in a proof will be numbered simply as $(n)$. 
1. Definitions and notation. For the definitions of an equipped probability space $\left(\Omega, \mathfrak{F}, P ; \mathfrak{F}_{z}, z \in \mathbf{R}_{+}^{2}\right)$, or briefly $\left(\Omega, \mathfrak{F}, P ; \mathfrak{F}_{z}\right)$, and the sub- $\sigma$-fields $\mathfrak{F}_{z}^{1} \equiv \mathfrak{F}_{s,}$, and $\mathfrak{F}_{z}^{2} \equiv \mathfrak{F}_{, t}$ of $\mathfrak{F}$ for $z=(s, t) \in \mathbf{R}_{+}^{2}$, we refer to [18, Definition 1.1]. We write $\mathfrak{M}\left(\mathfrak{F}_{z}\right), \mathfrak{M}^{w}\left(\mathfrak{F}_{z}\right)$ and $\mathfrak{M}^{s}\left(\mathfrak{F}_{z}\right)$ respectively for the linear spaces of equivalence classes of martingales $M$ with $\partial M=0$, weak martingales $M$ with $\partial M=0$ and strong martingales with respect to $\left\{\mathfrak{F}_{z}\right\}$ in the sense of R. Cairoli and J. B. Walsh [2]. A superscript 2 added to $\mathfrak{M}$ will signify the subspace of square integrable members and the subscript $c$ will signify the subspace of continuous ones. For the definition of the linear spaces $\mathfrak{L}_{p}\left(\mathfrak{F}_{z}\right), p \in[1, \infty)$, of equivalence classes of 2 -parameter stochastic processes, see [18, Definition 2.4]. Similarly we define $\mathfrak{L}_{\infty}\left(\mathfrak{F}_{z}\right)$ to be the linear space of equivalence classes of measurable $\left\{\mathfrak{F}_{z}\right\}$-adapted stochastic processes $\Phi$ on $\left(\Omega, \mathfrak{F}, P ; \mathfrak{F}_{z}\right)$ such that for every $T>0$ there exists $C_{T}>0$ satisfying

$$
|\Phi(z)| \leqslant C_{T} \text { for all } z \leqslant(T, T) \text { a.s. }
$$

For a 2-parameter stochastic process $X$ on a complete probability measure space $(\Omega, \mathfrak{F}, P)$, let $\mathfrak{R}$ be the collection of the null sets and define

$$
\begin{aligned}
& \mathfrak{S}_{X, z}=\sigma\{X(\zeta), \zeta \leqslant z\}, \quad \mathfrak{H}_{X, z}=\sigma\left(\mathfrak{S}_{X, z} \cup \mathfrak{R}\right), \\
& \mathfrak{F}_{X, z}=\bigcap_{\mathscr{E}>0} \mathfrak{S}_{X(s+\mathscr{E}, t+\mathscr{E})} \quad \text { for every } z=(s, t) \in \mathbf{R}_{+}^{2} .
\end{aligned}
$$

$\left\{\mathcal{F}_{X, z}, z \in \mathbf{R}_{+}^{2}\right\}$ will be called the standard filtration associated with $X$.

DEFINITION 1.1. By an $\left\{\mathfrak{F}_{z}\right\}$-Brownian motion we mean a 2-parameter stochastic process $B$ on an equipped probability space $\left(\Omega, \mathfrak{F}, P ; \mathfrak{F}_{z}\right)$ such that:

$1^{\circ} . B$ is $\left\{\mathfrak{F}_{z}\right\}$-adapted,

$2^{\circ}$. every sample function of $B$ is a continuous function on $\mathbf{R}_{+}^{2}$,

$3^{\circ}$. $B\left(\left(z, z^{\prime}\right]\right)$ is distributed by $N\left(0,\left(s^{\prime}-s\right)\left(t^{\prime}-t\right)\right)$ for every $z=(s, t)$ and $z^{\prime}=\left(s^{\prime}, t^{\prime}\right)$ such that $z<z^{\prime}$,

$4^{\circ} . B\left(\left(z, z^{\prime}\right]\right)$ and $\sigma\left(\mathfrak{F}_{z}^{1} \cup \mathfrak{F}_{z}^{2}\right)$ are independent for every $z$ and $z^{\prime}$ such that $z<z^{\prime}$.

REMARK 1.2. Consider the Wiener space $\left(W, \mathfrak{B}(W), m_{W}\right)$, where $W$ is the space of all continuous real valued functions on $\mathbf{R}_{+}^{2}, \mathfrak{B}(W)$ is the $\sigma$-field of subsets of $W$ generated by the cylinder sets in $W$ and $m_{W}$ is the Wiener measure on $\mathfrak{B}(W)$, concentrated on the elements of $W$ which vanish on $\partial \mathbf{R}_{+}^{2}$, having the value

$$
\begin{aligned}
m_{W}(C)=\left[(2 \pi)^{m n}\left\{\prod_{i=1}^{m}\left(s_{i}-s_{i-1}\right)\right\}^{n}\left\{\prod_{j=1}^{n}\left(t_{j}-t_{j-1}\right)\right\}^{m}\right]^{-1 / 2} \\
\times \int_{E_{1,1}}(m n) \int_{E_{m, n}} \exp \left[-\frac{1}{2} \sum_{i=1}^{m} \sum_{j=1}^{n}\left\{\left(s_{i}-s_{i-1}\right)\left(t_{j}-t_{j-1}\right)\right\}^{-1}\right. \\
\left.\times\left\{u_{i, j}-u_{i-1, j}-u_{i, j-1}+u_{i-1, j-1}\right\}^{2}\right] d u_{1,1} \cdots d u_{m, n}
\end{aligned}
$$

with $u_{0, j}=u_{i, 0}=0$ for the cylinder set $C$ in $W$ given by

$$
C=\left\{w \in W ; w\left(s_{i}, t_{j}\right) \in E_{i, j} \text { for } i=1,2, \ldots, m, j=1,2, \ldots, n\right\},
$$

where $0=s_{0}<s_{1}<\cdots<s_{m}, 0=t_{0}<t_{1}<\cdots<t_{n}$ and $E_{i, j} \in \mathfrak{B}(\mathbf{R})$. 
The space $W$ is a complete separable metric space with respect to the metric $\rho_{W}$ defined by

$$
\rho_{W}\left(w_{1}, w_{2}\right)=\sum_{k=1}^{\infty} 2^{-k}\left\{\max _{(s, t) \leqslant(k, k)}\left|w_{1}(s, t)-w_{2}(s, t)\right| \wedge 1\right\}
$$

for $w_{1}, w_{2} \in W$. Furthermore $\mathfrak{B}(W)$ is equal to the $\sigma$-algebra of the Borel sets in the metric topology generated by $\rho_{W}$.

If we define a 2-parameter stochastic process $B$ on the probability space $\left(W, \mathfrak{R}(W), m_{W}\right)$ by

$$
B(z, w)=w(z) \text { for }(z, w) \in \mathbf{R}_{+}^{2} \times W,
$$

then its probability distribution is $m_{W}$.

Let $\mathfrak{F}_{W}$ be the completion of $\mathfrak{B}(W)$ with respect to $m_{W}$ and let $\left\{\mathfrak{F}_{B, z}, z \in \mathbf{R}_{+}^{2}\right\}$ be the standard filtration associated with $B$ on the complete probability measure space $\left(W, \mathfrak{F}_{W}, m_{W}\right)$ as defined by (1.0). Then $\left(W, \mathfrak{F}_{W}, m_{W} ; \mathfrak{F}_{B, z}\right)$ is an equipped probability space and $B$ is an $\left\{\mathfrak{F}_{B, z}\right\}$-Brownian motion with $\partial B=0$ on it.

For the class $\mathbf{M}\left(\mathbf{R}_{+}^{2} \times W\right)$ of functions to which the coefficients $\alpha$ and $\beta$ of stochastic differential equation (0.1) belong, see [18, Definition 3.2].

REMARK 1.3. If $a$ is a measurable transformation of $(\mathbf{R}, \mathfrak{B}(\mathbf{R}))$ into $(\mathbf{R}, \mathfrak{B}(\mathbf{R}))$, then $\alpha$ defined by $\alpha(z, w)=a(w(z))$ for $(z, w) \in \mathbf{R}_{+}^{2} \times W$ belongs to the class $\mathbf{M}\left(\mathbf{R}_{+}^{2} \times W\right)$. (This is the type of coefficients in the stochastic differential equation (0.3).)

REMARK 1.4. Let $\alpha \in \mathbf{M}\left(\mathbf{R}_{+}^{2} \times W\right)$. Then for each fixed $z \in \mathbf{R}_{+}^{2}$

$$
\alpha(z, w(\cdot \wedge z))=\alpha(z, w) \text { for every } w \in W .
$$

In particular for every $w_{1}, w_{2} \in W$,

$$
w_{1}(\zeta)=w_{2}(\zeta) \text { for all } \zeta \leqslant z \Rightarrow \alpha\left(z, w_{1}\right)=\alpha\left(a, w_{2}\right)
$$

Proof. With $z \in \mathbf{R}_{+}^{2}$ fixed, consider the random vector $[w(\zeta), \zeta \leqslant z], w \in W$, on the measurable space $(W, \mathfrak{B}(W))$. Let $\left(\mathbf{R}^{[0, z]}, \mathfrak{B}^{[0, z]}\right)$ be the product measurable space $\prod_{\zeta \leqslant z}\left(\mathbf{R}_{\zeta}, \mathfrak{B}\left(\mathbf{R}_{\zeta}\right)\right)$. Since $\alpha(z, \cdot)$ is a measurable transformation of $\left(W, \mathfrak{B}_{z}(W)\right)$ into $\left(\mathbf{R}, \mathfrak{B}(\mathbf{R})\right.$ ) and since $\mathfrak{B}_{z}(W)$ is the sub- $\sigma$-field of $\mathfrak{B}(W)$ generated by the random vector $[w(\zeta), \zeta \leqslant z], w \in W$, there exists a measurable transformation $\varphi$ of $\left(\mathbf{R}^{[0, z]}, \mathfrak{B}^{[0, z]}\right)$ into $(\mathbf{R}, \mathfrak{B}(\mathbf{R}))$ such that

$$
\alpha(z, w)=\varphi([w(\zeta), \zeta \leqslant z]) \text { for } w \in W .
$$

Then

$$
\begin{aligned}
\alpha(z, w(\cdot \wedge z)) & =\varphi([w(\zeta \wedge z), \zeta \leqslant z]) \\
& =\varphi([w(\zeta), \zeta \leqslant z])=\alpha(z, w) \quad \text { for } w \in W
\end{aligned}
$$

DEFinition 1.5. Let $\alpha, \beta \in \mathbf{M}\left(\mathbf{R}_{+}^{2} \times W\right)$. By a solution of the stochastic differential equation

$$
X(z)=X(0)+\int_{R_{z}} \alpha(\zeta, X) d B_{\zeta}+\int_{R_{z}} \beta(\zeta, X) d \zeta \quad \text { for } z \in \mathbf{R}_{+}^{2}
$$


we mean a pair of 2-parameter stochastic processes $(X, B)$ on some equipped probability space $\left(\Omega, \mathfrak{F}, P ; \mathfrak{F}_{z}\right)$ satisfying the following conditions:

$1^{\circ} . B$ is an $\left\{\mathfrak{F}_{z}\right\}$-Brownian motion with $\partial B=0$,

$2^{\circ} . X$ is an $\left\{\mathfrak{F}_{z}\right\}$-adapted process whose sample functions are all continuous on $\mathbf{R}_{+}^{2}$,

$3^{\circ}$. the two 2-parameter stochastic processes $\Phi$ and $\Psi$ on $(\Omega, \mathfrak{F}, P)$ defined by

$$
\begin{array}{ll}
\Phi(z, \omega)=\alpha(z, X(\cdot, \omega)) & \text { for }(z, \omega) \in \mathbf{R}_{+}^{2} \times \Omega, \\
\Psi(z, \omega)=\beta(z, X(\cdot, \omega)) & \text { for }(z, \omega) \in \mathbf{R}_{+}^{2} \times \Omega
\end{array}
$$

are in $\mathfrak{Q}_{2}\left(\mathfrak{F}_{z}\right)$ and $\mathfrak{Q}_{1}\left(\mathfrak{F}_{z}\right)$ respectively,

$4^{\circ}$. with probability 1 , (1.1) holds for all $z \in \mathbf{R}_{+}^{2}$.

Consider the following continuity condition $(\mathrm{C} 1)$ and order of growth condition (C2) on our $\alpha, \beta \in \mathbf{M}\left(\mathbf{R}_{+}^{2} \times W\right)$ :

(C1) $\alpha$ and $\beta$ are jointly continuous in $z$ and $w$.

(C2) There exists a Borel measure $\lambda$ on $\mathbf{R}_{+}^{2}$ which is finite for every compact subset of $\mathbf{R}_{+}^{2}$ and satisfies the condition that for every $T>0$ there exists $L_{T}>0$ such that

$$
\alpha(z, w)^{2}+\beta(z, w)^{2} \leqslant L_{T}\left\{\int_{R_{z}} w(\zeta)^{2} \lambda(d \zeta)+w(z)^{2}+1\right\}
$$

for all $z \leqslant(T, T)$ and $w \in W$. Let us write for brevity

$$
\Lambda_{T} \equiv \lambda([0, T] \times[0, T]) .
$$

The objective of this article is to show that if $\alpha$ and $\beta$ satisfy (C1) and (C2), then (1.1) has a solution.

For the collection $\mathbf{L}_{2}^{c}\left(\mathfrak{F}_{z}\right)$ of 2-parameter stochastic processes, see [18, Definition 3.9].

REMARK 1.6. Let $\alpha, \beta \in \mathbf{M}\left(\mathbf{R}_{+}^{2} \times W\right)$ and let $X$ be an $\left\{\mathfrak{F}_{z}\right\}$-adapted 2-parameter stochastic process on an equipped probability space $\left(\Omega, \mathfrak{F}, P\right.$; $\left.\mathfrak{F}_{z}\right)$ whose sample functions are all continuous on $\mathbf{R}_{+}^{2}$. Then $\Phi$ and $\Psi$ defined by (1.2) and (1.3) are $\left\{\mathfrak{T}_{z}\right\}$-adapted measurable processes on $\left(\Omega, \mathfrak{F}, P ; \mathfrak{F}_{z}\right)$. If in addition $\alpha$ and $\beta$ satisfy the order of growth condition $(\mathrm{C} 2)$ and $X$ is in $\mathbf{L}_{2}^{c}\left(\mathfrak{F}_{z}\right)$, then $\Phi, \Psi \in \mathfrak{L}_{2}\left(\mathfrak{F}_{z}\right)$.

\section{Solution of the stochastic differential equation with stepwise coefficients.}

Definition 2.1. Let $\mathbf{M}_{0}\left(\mathbf{R}_{+}^{2} \times W\right)$ be the collection of all $\alpha \in \mathbf{M}\left(\mathbf{R}_{+}^{2} \times W\right)$ satisfying the additional condition that there exist $0=s_{0}<s_{1}<\cdots$ with $\lim _{i \rightarrow \infty} s_{i}$ $=\infty$ and $0=t_{0}<t_{1}<\cdots$ with $\lim _{j \rightarrow \infty} t_{j}=\infty$ such that with $z_{i, j}=\left(s_{i}, t_{j}\right)$ for $i, j=0,1,2, \ldots$, we have $\alpha(z, w)=\alpha\left(z_{i, j}, w\right)$ for $z \in\left[z_{i, j}, z_{i+1, j+1}\right)$ for $i, j=$ $0,1,2, \ldots$, and $w \in W$.

The following theorem is a modification of the standard one-parameter proof. See C. Doléans-Dade and P. A. Meyer [4], J. Jacod and J. Memin [7] or S. Watanabe [16].

THEOREM 2.2. Let $\left(\Omega, \mathfrak{F}, P ; \mathfrak{F}_{z}\right)$ be an equipped probability space on which an $\left\{\hat{\imath}_{z}\right\}$-Brownian motion $B$ with $\partial B=0$ exists. Let $\alpha, \beta \in \mathbf{M}_{0}\left(\mathbf{R}_{+}^{2} \times W\right)$ and assume that $\alpha$ and $\beta$ satisfy the order of growth condition (C2) in $\S 1$. Then for every 
$\mathfrak{\Upsilon}_{0}-$-measurable random variable $\xi$ on $(\Omega, \mathfrak{F}, P)$ with $\mathbf{E}\left[\xi^{2}\right]<\infty$ there exists a 2parameter stochastic process $X$ on $(\Omega, \mathfrak{F}, P)$ such that

$$
\begin{gathered}
X \in \mathbf{L}_{2}^{c}\left(\mathfrak{F}_{z}\right), \\
X(0)=\xi
\end{gathered}
$$

and $(X, B)$ is a solution of the stochastic differential equation (1.1), i.e.,

$$
X(z)=X(0)+\int_{R_{z}} \alpha(\zeta, X) d B_{\zeta}+\int_{R_{z}} \beta(\zeta, X) d \zeta \quad \text { for } z \in \mathbf{R}_{+}^{2} .
$$

Proof. Let us define a 2-parameter stochastic process $X_{0,0}$ on $(\Omega, \mathfrak{F}, P)$ by setting

$$
X_{0,0}(z, \omega)=\xi(\omega) \quad \text { for }(z, \omega) \in \mathbf{R}_{+}^{2} \times \Omega .
$$

Note that $X_{0,0} \in \mathbf{L}_{2}^{c}\left(\mathfrak{F}_{z}\right)$ trivially.

Next, let us define a 2-parameter stochastic process $X_{1,1}$ on $(\Omega, \mathfrak{F}, P)$ by setting (2)

$$
X_{1.1}(z)= \begin{cases}X_{0,0}(0)+\int_{R_{z}} \alpha\left(0, X_{0,0}\right) d B_{\zeta}+\int_{R_{z}} \beta\left(0, X_{0,0}\right) d \zeta & \text { for } z \in R_{z_{1.1}}, \\ X_{1,1}\left(z \wedge z_{1,1}\right) & \text { for } z \in \mathbf{R}_{+}^{2}-R_{z_{1,1}},\end{cases}
$$

where the two stochastic integrals exist according to Remark 1.6 since $X_{0,0} \in \mathbf{L}_{2}^{c}\left(\mathfrak{F}_{z}\right)$. Our $X_{1,1}$ has the following properties:

$$
\begin{cases}X_{1,1}\left(z \wedge z_{1,1}\right)=X_{1,1}(z) & \text { for } z \in \mathbf{R}_{+}^{2}-R_{z_{1,1}} \\ X_{1,1} \in \mathbf{L}_{2}^{c}\left(\mathfrak{F}_{z}\right), & \\ X_{1,1}(0)=\xi, & \text { satisfies (2.3) on } R_{z_{1,1}} \\ X_{1,1} & \end{cases}
$$

The first and the third property in (3) are obvious from (2). For the left side of (2) for $z \in R_{z_{1.1}}$ we have by (1.4) and (1.5)

$$
\sup _{z \leqslant-_{1.1}} \mathbf{E}\left[X_{1,1}(z)^{2}\right] \leqslant 3^{2}\left\{\mathbf{E}\left[\xi^{2}\right]+\left(1+s_{1} t_{1}\right) s_{1} t_{1} L_{0}\left\{\left(\Lambda_{0}+1\right) \mathbf{E}\left[\xi^{2}\right]+1\right\}\right\}<\infty .
$$

This, together with the way $X_{1,1}$ is defined on $\mathbf{R}_{+}^{2}-R_{z_{1.1}}$ by (2), implies that $X_{1,1} \in \mathbf{L}_{2}^{c}\left(\mathfrak{F}_{z}\right)$. Then according to Remark 1.6, the stochastic integrals $\int_{R_{z}} \alpha\left(\zeta, X_{1,1}\right) d B_{\zeta}$ and $\int_{R_{z}} \beta\left(\zeta, X_{1,1}\right) d \zeta$ exist for $z \in \mathbf{R}_{+}^{2}$. It remains to show that $X_{1,1}$ satisfies (2.3) on $R_{z_{1,1}}$. Recalling $X_{1,1}(0)=X_{0,0}(0)$ we have by Remark 1.4 and then by Definition 2.1,

$$
\left\{\begin{array}{l}
\alpha\left(0, X_{0,0}\right)=\alpha\left(0, X_{1,1}\right)=\alpha\left(\zeta, X_{1,1}\right) \\
\beta\left(0, X_{0,0}\right)=\beta\left(0, X_{1,1}\right)=\beta\left(\zeta, X_{1,1}\right)
\end{array} \quad \text { for } \zeta \in\left[0, z_{1,1}\right) .\right.
$$

Then from (2) and (4) we have

$$
X_{1,1}(z)=X_{1,1}(0)+\int_{R_{z}} \alpha\left(\zeta, X_{1,1}\right) d B_{\zeta}+\int_{R_{z}} \beta\left(\zeta, X_{1,1}\right) d \zeta \quad \text { for } z \in R_{z_{1,1}},
$$

i.e., $X_{1,1}$ satisfies (2.3) on $R_{z_{1.1}}$. This completes the verification of (3). 
Next, suppose that for some $(i, j)$ there exists a 2-parameter stochastic process $X_{i, j}$ on $(\Omega, \mathfrak{F}, P)$ with the following properties:

$$
\begin{cases}X_{i, j}\left(z \wedge z_{i, j}\right)=X_{i, j}(z) & \text { for } z \in \mathbf{R}_{+}^{2}-R_{z_{i, j}} \\ X_{i, j} \in \mathbf{L}_{2}^{c}\left(\mathfrak{F}_{z}\right) & \\ X_{i, j}(0)=\xi, & \text { satisfies (2.3) on } R_{z_{i, j}} .\end{cases}
$$

We proceed to show that under the assumption of existence of such $X_{i, j}$ there exists a 2-parameter stochastic process $X_{i+1, j}$ on $(\Omega, \mathfrak{F}, P)$ such that

$$
\begin{cases}X_{i+1, j}(z)=X_{i, j}(z) & \text { for } z \in R_{z_{i, j}} \\ X_{i+1, j}\left(z \wedge z_{i+1, j}\right)=X_{i+1, j}(z) & \text { for } z \in \mathbf{R}_{+}^{2}-R_{z_{i+1, j}} \\ X_{i+1, j} \in \mathbf{L}_{2}^{c}\left(\mathfrak{F}_{z}\right), & \\ X_{i+1, j} & \text { satisfies (2.3) on } R_{z_{i+1, j}}\end{cases}
$$

To construct such a process $X_{i+1, j}$ let us define $X_{i+1, j}$ on $R_{z_{i, j}}$ and on $\mathbf{R}_{+}^{2}-R_{z_{i+1, j}}$ by the first and the second equality in (6) respectively and set

$$
\begin{aligned}
X_{i+1, j}(z)= & X_{i, j}\left(s_{i}, t\right)+\int_{\left[s_{i}, s\right] \times[0, t]} \alpha\left(s_{i}, v, X_{i, j}\right) d B_{\zeta} \\
& +\int_{\left[s_{i}, s\right] \times[0, t]} \beta\left(s_{i}, v, X_{i, j}\right) d \zeta
\end{aligned}
$$

for $z=(s, t) \in\left[s_{i}, s_{i+1}\right] \times\left[0, t_{j}\right]$ and $\zeta=(u, v) \leqslant z$. The fact that $X_{i+1, j}$ thus defined is in $\mathbf{L}_{2}^{c}\left(\mathfrak{F}_{z}\right)$ can be verified in the same way as we did for $X_{1,1}$ above by using the assumption in (5) that $X_{i, j}$ is in $\mathbf{L}_{2}^{c}\left(\mathfrak{F}_{z}\right)$ and by (1.4). To verify that $X_{i+1, j}$ satisfies (2.3) on $R_{z_{i+1 . j}}$, observe first that by (7),

$$
X_{i+1, j}\left(s_{i}, t\right)=X_{i, j}\left(s_{i}, t\right) \quad \text { for } t \in\left[0, t_{j}\right] .
$$

By the first equality in (6) and by Remark 1.4,

$$
\alpha\left(z, X_{i+1, j}\right)=\alpha\left(z, X_{i, j}\right) \text { and } \beta\left(z, X_{i+1, j}\right)=\beta\left(z, X_{i, j}\right) \quad \text { for } z \in R_{z_{i, j}} .
$$

These equalities and the assumption that $X_{i, j}$ satisfies (2.3) on $R_{z_{i, j}}$ imply that $X_{i+1, j}$ too satisfies (2.3) on $R_{z_{i, j}}$. Next, consider $z=(s, t) \in\left[s_{i}, s_{i+1}\right] \times\left[0, t_{j}\right]$. Let us write

$$
\begin{aligned}
X_{i+1, j}(z)-X_{i+1, j}(0)= & \left\{X_{i+1, j}(z)-X_{i+1, j}\left(s_{i}, t\right)\right\} \\
& +\left\{X_{i+1, j}\left(s_{i}, t\right)-X_{i+1, j}(0)\right\} .
\end{aligned}
$$

Now since $X_{i+1, j}$ satisfies (2.3) on $R_{z_{i, j}}$ we have

$$
\begin{aligned}
X_{i+1, j}\left(s_{i}, t\right)-X_{i+1, j}(0)= & \int_{R_{s_{i}, t}} \alpha\left(\zeta, X_{i+1, j}\right) d B_{\zeta} \\
& +\int_{R_{s_{i}, t}} \beta\left(\zeta, X_{i+1, j}\right) d \zeta \quad \text { for } t \in\left[0, t_{j}\right] .
\end{aligned}
$$


On the other hand from (8) and (7) we have for $z=(s, t) \in\left[s_{i}, s_{i+1}\right] \times\left[0, t_{j}\right]$ and $\zeta=(u, v) \leqslant z$,

$$
\begin{aligned}
X_{i+1, j}(z)- & X_{i+1, j}\left(s_{i}, t\right)=X_{i+1, j}(z)-X_{i, j}\left(s_{i}, t\right) \\
& =\int_{\left[s_{i}, s\right] \times[0, t]} \alpha\left(s_{i}, v, X_{i, j}\right) d B_{\zeta}+\int_{\left[s_{i}, s\right] \times[0, t]} \beta\left(s_{i}, v, X_{i, j}\right) d \zeta .
\end{aligned}
$$

The fact that $X_{i+1, j}=X_{i, j}$ on $R_{z_{i, j}}$ implies according to Remark 1.4

$$
\begin{aligned}
\alpha\left(s_{i}, v, X_{i, j}\right) & =\alpha\left(s_{i}, v, X_{i+1, j}\right) \\
& =\alpha\left(u, v, X_{i+1, j}\right) \quad \text { for }(u, v) \in\left[s_{i}, s_{i+1}\right) \times\left[0, t_{j}\right] .
\end{aligned}
$$

By (12) and a similar expression for $\beta$ we can rewrite (11) as

$$
\begin{aligned}
X_{i+1, j}(z)-X_{i+1, j}\left(s_{i}, t\right)= & \int_{\left[s_{i}, s\right] \times[0, t]} \alpha\left(\zeta, X_{i+1, j}\right) d B_{\zeta} \\
& +\int_{\left[s_{i}, s\right] \times[0, t]} \beta\left(\zeta, X_{i+1, j}\right) d \zeta
\end{aligned}
$$

for $z=(s, t) \in\left[s_{i}, s_{i+1}\right] \times\left[0, t_{j}\right]$ and $\zeta=(u, v) \leqslant z$. Using (10) and (13) in (9) we have

$$
\begin{aligned}
X_{i+1, j}(z)-X_{i+1, j}(0)= & \int_{R_{z}} \alpha\left(\zeta, X_{i+1, j}\right) d B_{\zeta} \\
& +\int_{R_{z}} \beta\left(\zeta, X_{i+1, j}\right) d \zeta \quad \text { for } z \in\left[s_{i}, s_{i+1}\right] \times\left[0, t_{j}\right] .
\end{aligned}
$$

Thus $X_{i+1, j}$ satisfies (2.3) on $\left[s_{i}, s_{i+1}\right] \times\left[0, t_{j}\right]$. Since $X_{i+1, j}$ satisfies (2.3) on $R_{z_{i, j}}$ as we saw above, $X_{i+1, j}$ satisfies (2.3) on $R_{z_{i+1, j}}$. This completes the verification of (6) for our $X_{i+1, j}$.

Similarly under the assumption of the existence of a 2-parameter stochastic process $X_{i, j}$ on $(\Omega, \mathfrak{F}, P)$ having the properties (5) we can show the existence of a 2-parameter stochastic process $X_{i, j+1}$ on $(\Omega, \mathfrak{F}, P)$ such that

$$
\begin{cases}X_{i, j+1}(z)=X_{i, j}(z) & \text { for } z \in R_{z_{i, j}}, \\ X_{i, j+1}\left(z \wedge z_{i, j+1}\right)=X_{i, j}(z) & \text { for } z \in \mathbf{R}_{+}^{2}-R_{z_{i, j+1}}, \\ X_{i, j+1} \in \mathbf{L}_{2}^{c}\left(\mathfrak{F}_{z}\right), & \\ X_{i, j+1} & \text { satisfies (2.3) on } R_{z_{i, j+1}}\end{cases}
$$

Now starting with $X_{1,1}$ constructed by (2) and having the properties (3), we obtain by using (6) and (14) alternatingly, each step being justified by verification of (5), a sequence of 2-parameter stochastic processes

$$
X_{1,1}, X_{2,1}, X_{2,2}, \ldots, X_{n, n-1}, X_{n, n}, X_{n+1, n}, X_{n+1, n+1}, \ldots
$$


on $(\Omega, \mathcal{T}, P)$ having the properties:

$$
\begin{cases}X_{i, j}(z)=X_{p, q}(z) & \text { for } z \in R_{z_{p, 4}} \text { when } p \leqslant i \text { and } q \leqslant j, \\ X_{i, j} \in \mathbf{L}_{2}^{c}\left(\tilde{z}_{z}\right), & \\ X_{i, j}(0)=\xi, & \text { satisfies (2.3) on } R_{z_{i, j}}\end{cases}
$$

Let a 2-parameter stochastic process $X$ on $(\Omega, \mathfrak{F}, P)$ be defined by setting $X=X_{i, j}$ on $R_{z, 1}$ as $(i, j)$ runs through the sequence $(1,1),(2,1),(2,2), \ldots,(n, n-1),(n, n)$, $(n+1, n),(n+1, n+1), \ldots$ Thus defined, $X$ satisfies (2.2) and (2.3). The fact that $X \in \mathbf{L}_{2}^{c}(\mathfrak{z})$ follows from the fact that $X_{i, j} \in \mathbf{L}_{2}^{c}\left(\widetilde{F}_{z}\right)$ for $(i, j)=(1,1),(2,1),(2,2)$, $(3,2),(3,3), \ldots$

3. The uniform Kolmogorov condition for the family of solutions of the approximating stochastic differential systems with stepwise coefficients. For $l=1,2, \ldots$, let $[\cdot]_{l}$ be a step function on $[0, \infty)$ defined by $[s]_{l}=i 2^{-l}$ for $s \in\left[i 2^{-l},(i+1) 2^{-l}\right)$, $i=0,1,2, \ldots$ We write $[z]_{l}=\left([s]_{l},[t]_{l}\right)$ for $z=(s, t) \in \mathbf{R}_{+}^{2}$. With $\alpha \in \mathbf{M}\left(\mathbf{R}_{+}^{2} \times W\right)$, we define $\alpha_{l} \in \mathbf{M}_{0}\left(\mathbf{R}_{+}^{2} \times W\right)$ by

$$
\alpha_{l}(z, w)=\alpha\left([z]_{l}, w\right) \text { for }(z, w) \in \mathbf{R}_{+}^{2} \times W .
$$

Let $\left(\Omega, \mathfrak{F}, P ; \mathfrak{F}_{z}\right)$ be an equipped probability space on which an $\left\{\mathfrak{F}_{z}\right\}$-Brownian motion $B$ with $\partial B=0$ exists. Let $\alpha, \beta \in \mathbf{M}\left(\mathbf{R}_{+}^{2} \times W\right)$ and assume that $\alpha$ and $\beta$ satisfy the order of growth condition (C2) in $\S 1$. For $l=1,2, \ldots$, let $\alpha$, and $\beta$, be defined by (3.1). Then by Theorem 2.2 for every $\tilde{F}_{0}$-measurable random variable $\xi$ on $(\Omega, \tilde{i}, P)$ with $\mathbf{E}\left[\xi^{2}\right]<\infty$, there exists a 2-parameter stochastic process $X_{l}$ on $(\Omega, \tilde{i}, P)$ such that

$$
\begin{gathered}
X_{l} \in \mathbf{L}_{2}^{c}\left(\tilde{\digamma}_{z}\right), \\
X_{l}(0)=\xi \\
X_{l}(z)=X_{l}(0)+\int_{R_{z}} \alpha_{l}\left(\zeta, X_{l}\right) d B_{\zeta}+\int_{R_{z}} \beta_{l}\left(\zeta, X_{l}\right) d \zeta
\end{gathered}
$$

for $z \in \mathbf{R}_{+}^{2}$ and $l=1,2, \ldots$

In order to show that the solutions $X_{l}$ of the stochastic differential systems (3.3) and (3.4) with stepwise coefficients $\alpha_{l}$ and $\beta$, for $l=1,2, \ldots$ approximating the coefficients $\alpha$ and $\beta$ in (0.1) and (0.2) satisfy the uniform Kolmogorov condition $(0.5)$, we obtain first uniform estimates $(0.4)$ for the moments of $X_{l}, l=1,2, \ldots$

Proposition 3.1. Let $\alpha$, and $\beta, l=1,2, \ldots$, be defined by (3.1) with $\alpha, \beta \in$ $\mathbf{M}\left(\mathbf{R}_{+}^{2} \times W\right)$ satisfying the condition $(\mathrm{C} 2)$ in $\$ 1$. Let $X_{l}$ be a solution for the differential system (3.3) and (3.4) for $l=1,2, \ldots$ Then for every positive integer $n=2$ or $n \geqslant 4$ and every $T>0$ there exists a constant $C_{n, T} \geqslant 1$, independent of $l=1,2, \ldots$, such that

$$
\mathbf{E}\left[\left|X_{l}(z)\right|^{n}\right] \leqslant\left\{1+\mathbf{E}\left[|\xi|^{n}\right]\right\} e^{2 C_{n, T} s t}-1
$$

for $z=(s, t) \leqslant(T, T)$ and $l=1,2, \ldots$ 
An estimate of the form (3.5) for a solution $X$ of the stochastic differential system (0.1) and (0.2) with coefficients $\alpha, \beta \in \mathbf{M}\left(\mathbf{R}_{+}^{2} \times W\right)$ satisfying (C2) was proved by $\mathrm{J}$. Reid (see [11, Theorem 4.2]). By modifying his proof the existence of $C_{n, T} \geqslant 1$ satisfying (3.5) for all $l=1,2, \ldots$ can be shown. The proof for this is lengthy but not difficult and will not be presented here.

Proposition 3.2. Let $X_{l}$ be as in Proposition 3.1 for $l=1,2, \ldots$ Assume further that $\mathbf{E}\left[\xi^{6}\right]<\infty$. Then for every $T>0$ there exists $\Gamma_{T}>0$ such that

$$
\mathbf{E}\left[\left|X_{l}(z)-X_{l}\left(z^{\prime}\right)\right|^{6}\right] \leqslant \Gamma_{T} d\left(z, z^{\prime}\right)^{3}
$$

for $z, z^{\prime} \leqslant(T, T)$ and $l=1,2, \ldots$

Proof. Let $T>0$ be fixed. For $z, z^{\prime} \leqslant(T, T)$, where $z=(s, t)$ and $z^{\prime}=\left(s^{\prime}, t^{\prime}\right)$, let us introduce the following notations:

$$
\left\{\begin{array}{l}
s_{1}=s \wedge s^{\prime}, \quad s_{2}=s \vee s^{\prime}, \quad t_{1}=t \wedge t^{\prime}, \quad t_{2}=t \vee t^{\prime} \\
z_{1}=z \wedge z^{\prime}=\left(s_{1}, t_{1}\right), \quad z_{2}=z \vee z^{\prime}=\left(s_{2}, t_{2}\right), \\
\Delta_{1}=\left[s_{1}, s_{2}\right] \times\left[0, t_{1}\right], \quad \Delta_{2}=\left[0, s_{1}\right] \times\left[t_{1}, t_{2}\right], \quad \Delta_{3}=\left[s_{1}, s_{2}\right] \times\left[t_{1}, t_{2}\right]
\end{array}\right.
$$

From (3.3) and (3.4) we have

$$
\left|X_{l}(z)-X_{l}\left(z^{\prime}\right)\right| \leqslant \sum_{i=1}^{3}\left\{\left|I_{k}\left(\alpha_{l}\right)\right|+\left|J_{k}\left(\beta_{l}\right)\right|\right\}
$$

where we set

(3) $I_{k}\left(\alpha_{l}\right)=\int_{\Delta_{k}} \alpha_{l}\left(\zeta, X_{l}\right) d B_{\zeta}$ and $J_{k}\left(\beta_{l}\right)=\int_{\Delta_{k}} \beta_{l}\left(\zeta, X_{l}\right) d \zeta \quad$ for $k=1,2,3$.

Let $I_{l, N}, a_{l, N}$ and $b_{l, N}$ be defined by

$$
I_{l, N}(z, \omega)=\left\{\begin{array}{ll}
1 & \text { when } \sup _{\zeta \leqslant z}\left|X_{l}(\zeta, \omega)\right| \leqslant N \\
0 & \text { otherwise }
\end{array} \text { for }(z, \omega) \in \mathbf{R}_{+}^{2} \times \Omega\right.
$$

and

$$
\left\{\begin{array}{l}
a_{l, N}(z, \omega)=I_{l, N}(z, \omega) \alpha_{l}\left(z, X_{l}(\cdot, \omega)\right) \\
b_{l, N}(z, \omega)=I_{l, N}(z, \omega) \beta_{l}\left(z, X_{l}(\cdot, \omega)\right)
\end{array} \quad \text { for }(z, \omega) \in \mathbf{R}_{+}^{2} \times \Omega .\right.
$$

Then by (2) and (3)

$$
\left|X_{l}(z)-X_{l}\left(z^{\prime}\right)\right| I_{l, N}\left(z_{2}\right) \leqslant \sum_{k=1}^{3}\left\{\left|I_{k}\left(a_{l, N}\right)\right|+\left|J_{k}\left(b_{l, N}\right)\right|\right\},
$$

where we set

(5) $\quad I_{k}\left(a_{l, N}\right)=\int_{\Delta_{k}} a_{l, N}(\zeta) d B_{\zeta}$ and $J_{k}\left(b_{l, N}\right)=\int_{\Delta_{k}} b_{l, N}(\zeta) d \zeta \quad$ for $k=1,2,3$.

Thus

(6) $\mathbf{E}\left[\left|X_{l}(z)-X_{l}\left(z^{\prime}\right)\right|^{6} I_{l, N}\left(z_{2}\right)\right] \leqslant 6^{6} \sum_{k=1}^{3}\left\{\mathbf{E}\left[I_{k}\left(a_{l, N}\right)^{6}\right]+\mathbf{E}\left[J_{k}\left(b_{l, N}\right)^{6}\right]\right\}$. 
Now if $\Phi \in \mathfrak{Q}_{\infty}\left(\mathfrak{F}_{z}\right), z, z_{0} \in \mathbf{R}_{+}^{2}, \Delta=\left[z_{0}, z_{0}+z\right]$ and $\tau$ is a translation in $\mathbf{R}_{+}^{2}$ by $z_{0}$, i.e., $\tau(\zeta)=z_{0}+\zeta$ for $\zeta \in \mathbf{R}_{+}^{2}$, then

$$
\mathbf{E}\left[\left\{\int_{\Delta} \Phi(\zeta) d B_{\zeta}\right\}^{6}\right] \leqslant \gamma m_{L}(\Delta)^{2} \mathbf{E}\left[\int_{\tau^{-1}(\Delta)} \Phi(\tau(\zeta))^{6} d \zeta\right]
$$

where $\gamma>0$ is an absolute constant and $m_{L}$ is the Lebesgue measure on $\mathbf{R}_{+}^{2}$. (See Theorem 2.1 in Guyon and Prum [5].) If we define three translations $\tau_{1}, \tau_{2}$ and $\tau_{3}$ in $\mathbf{R}_{+}^{2}$ by $\tau_{1}(\zeta)=\left(s_{1}+u, v\right), \tau_{2}(\zeta)=\left(u, t_{1}+v\right)$ and $\tau_{3}(\zeta)=\left(s_{1}+u, t_{1}+v\right)$ for $\zeta=$ $(u, v) \in \mathbf{R}_{+}^{2}$ with $\tau_{1}^{-1}\left(\Delta_{1}\right)=\left[0, s_{2}-s_{1}\right] \times\left[0, t_{1}\right], \tau_{2}^{-1}\left(\Delta_{2}\right)=\left[0, s_{1}\right] \times\left[0, t_{2}-t_{1}\right]$ and $\tau_{3}^{-1}\left(\Delta_{3}\right)=\left[0, s_{2}-s_{1}\right] \times\left[0, t_{2}-t_{1}\right]$, then by (7) we have

$$
\mathbf{E}\left[I_{k}\left(a_{l, N}\right)^{6}\right] \leqslant \gamma m_{L}\left(\Delta_{k}\right)^{2} \mathbf{E}\left[\int_{\tau_{k}^{-1}\left(\Delta_{k}\right)} \alpha_{l}\left(\tau_{k}(\zeta), X_{l}\right)^{6} d \zeta\right]
$$

for $k=1,2,3$. Regarding $\mathbf{E}\left[J_{k}\left(b_{l, N}\right)^{6}\right]$, we have by Hölder's inequality

$$
\left|\int_{\Delta_{k}} b_{I, N}(\zeta) d \zeta\right| \leqslant m_{L}\left(\Delta_{k}\right)^{5 / 6}\left\{\int_{\Delta_{k}} b_{I, N}(\zeta)^{6} d \zeta\right\}^{1 / 6}
$$

and therefore by the translation invariance of $m_{L}$ and then by the definition of $b_{I, N}$

$$
\mathbf{E}\left[J_{k}\left(b_{l, N}\right)^{6}\right] \leqslant m_{L}\left(\Delta_{k}\right)^{5} \mathbf{E}\left[\int_{\tau_{k}{ }^{1}\left(\Delta_{k}\right)} \beta_{l}\left(\tau_{k}(\zeta), X_{l}\right)^{6} d \zeta\right]
$$

for $k=1,2,3$. Using (8) and (9) in (6), letting $n \rightarrow \infty$ on the left side of (6) and recalling $\lim _{N \rightarrow \infty} I_{l, N}\left(z_{2}\right)=1$ by the continuity of the sample functions of $X_{l}$, we have

$$
\begin{aligned}
\mathbf{E}\left[\left|X_{l}(z)-X_{l}\left(z^{\prime}\right)\right|^{6}\right] \leqslant & 6^{6} \sum_{k=1}^{3} m_{L}\left(\Delta_{k}\right)^{2}\left\{\gamma+m_{L}\left(\Delta_{k}\right)^{3}\right\} \\
& \times \int_{\tau_{h}^{-1}\left(\Delta_{k}\right)} \mathbf{E}\left[\alpha_{l}\left(\tau_{k}(\zeta), X_{l}\right)^{6}+\beta_{l}\left(\tau_{k}(\zeta), X_{l}\right)^{6}\right] d \zeta .
\end{aligned}
$$

For $\zeta \in \tau_{k}^{-1}\left(\Delta_{k}\right)$, we have $\tau_{k}(\zeta) \in \Delta_{k} \subset[0, T] \times[0, T]$. Therefore by (3.1) and (1.4) we have

$$
\begin{aligned}
& \alpha_{l}\left(\tau_{k}(\zeta), X_{l}\right)^{6}+\beta_{l}\left(\tau_{k}(\zeta), X_{l}\right)^{6} \\
& \quad \leqslant\left\{\alpha_{l}\left(\tau_{k}(\zeta), X_{l}\right)^{2}+\beta_{l}\left(\tau_{k}(\zeta), X_{l}\right)^{2}\right\}^{3} \\
& \quad \leqslant L_{T}^{3}\left\{1+X_{l}\left(\left[\tau_{k}(\zeta)\right]_{l}\right)^{2}+\int_{[0, T] \times[0, T]} X_{l}(\zeta)^{2} \lambda(d \zeta)\right\}^{3}
\end{aligned}
$$

for $\zeta \in \tau_{k}^{-1}\left(\Delta_{k}\right)$. In expanding the cubic power in the last member of (11) we obtain finitely many terms, the expectation of each of which is bounded by a positive number which depends only on $T, L_{T}, \Lambda_{T}$ in (1.4) and (1.5), $\mathbf{E}\left[\xi^{6}\right], C_{2, T}, C_{4, T}$ and 
$C_{6, T}$ in Proposition 3.1. Let us show the calculation for just one term as an example. Thus for instance

$$
\begin{aligned}
& \mathbf{E}\left[X_{l}\left(\left[\tau_{k}(\zeta)\right]_{l}\right)^{2}\left\{\int_{[0, T] \times[0, T]} X_{l}(\zeta)^{2} \lambda(d \zeta)\right\}^{2}\right] \\
& \quad=\mathbf{E}\left[X_{l}\left(\left[\tau_{k}(\zeta)\right]_{l}\right)^{2} \int_{[0, T]^{4}} X_{l}\left(\zeta^{\prime}\right)^{2} X_{l}\left(\zeta^{\prime \prime}\right)^{2}(\lambda \times \lambda)\left(d\left(\zeta^{\prime}, \zeta^{\prime \prime}\right)\right)\right] \\
& \quad=\int_{[0, T]^{4}} \mathbf{E}\left[X_{l}\left(\left[\tau_{k}(\zeta)\right]_{l}\right)^{2} X_{l}\left(\zeta^{\prime}\right)^{2} X_{l}\left(\zeta^{\prime \prime}\right)^{2}\right](\lambda \times \lambda)\left(d\left(\zeta^{\prime}, \zeta^{\prime \prime}\right)\right) \\
& \quad \leqslant \int_{[0, T]^{4}} \mathbf{E}\left[X_{l}\left(\left[\tau_{k}(\zeta)\right]_{l}\right)^{6}\right]^{1 / 3} \mathbf{E}\left[X_{l}\left(\zeta^{\prime}\right)^{6}\right]^{1 / 3} \mathbf{E}\left[X_{l}\left(\zeta^{\prime \prime}\right)^{6}\right]^{1 / 3}(\lambda \times \lambda)\left(d\left(\zeta^{\prime}, \zeta^{\prime \prime}\right)\right) \\
& \quad \leqslant\left[\left\{1+\mathbf{E}\left[\xi^{6}\right]\right\} \exp \left(2 C_{6, T} T^{2}\right)-1\right] \Lambda_{T}^{2}
\end{aligned}
$$

by (3.5) in Proposition 3.1. Similar estimates hold for the other terms in the expansion of the cubic power in (11). Therefore there exists a positive number $K_{T}$ depending only on $T, L_{T}, \Lambda_{T}, \mathbf{E}\left[\xi^{6}\right], C_{2, T}, C_{4, T}$ and $C_{6, T}$ such that

$$
\mathbf{E}\left[\alpha_{l}\left(\tau_{k}(\zeta), X_{l}\right)^{6}+\beta_{l}\left(\tau_{k}(\zeta), X_{l}\right)^{6}\right] \leqslant K_{T} \text { for } \zeta \in \tau_{k}^{-1}\left(\Delta_{k}\right)
$$

Using (12) in (10) we have

$$
\begin{aligned}
\mathbf{E}\left[\left|X_{l}(z)-X_{l}\left(z^{\prime}\right)\right|^{6}\right] \leqslant 6{ }^{6} K_{T} \sum_{k=1}^{3} m_{L}\left(\Delta_{k}\right)^{2}\left\{\gamma+m_{L}\left(\Delta_{k}\right)^{3}\right\} m_{L}\left(\tau_{k}^{-1}\left(\Delta_{k}\right)\right) \\
\leqslant 6{ }^{6} K_{T}\left(\gamma+T^{6}\right) \sum_{k=1}^{3} m_{L}\left(\Delta_{k}\right)^{3} \quad \text { for } z, z^{\prime} \leqslant(T, T) \text { and } l=1,2, \ldots
\end{aligned}
$$

From (1)

$$
m_{L}\left(\Delta_{1}\right)^{3}=\left(s_{2}-s_{1}\right)^{3} t_{1}^{3}, \quad m_{L}\left(\Delta_{2}\right)^{3}=s_{1}^{3}\left(t_{2}-t_{1}\right)^{3}
$$

and

$$
m_{L}\left(\Delta_{3}\right)^{3}=\left(s_{2}-s_{1}\right)^{3}\left(t_{2}-t_{1}\right)^{3}
$$

and thus

$$
\begin{aligned}
m_{L}\left(\Delta_{k}\right)^{3} & \leqslant\left\{\left(s_{2}-s_{1}\right)^{2}+\left(t_{2}-t_{1}\right)^{2}\right\}^{3 / 2} T^{3} \\
& =T^{3} d\left(z, z^{\prime}\right)^{3} \quad \text { for } k=1,2,3
\end{aligned}
$$

Therefore

$$
\mathbf{E}\left[\left|X_{l}(z)-X_{l}\left(z^{\prime}\right)\right|^{6}\right] \leqslant \Gamma_{T} d\left(z, z^{\prime}\right)^{3} \quad \text { for } z, z^{\prime} \leqslant(T, T) \text { and } l=1,2, \ldots,
$$

where $\Gamma_{T}=3 \cdot 6{ }^{6} T^{3} K_{T}\left(\gamma+T^{6}\right)$. This completes the proof of (3.6). 
4. The existence theorem for solutions to the stochastic differential equations with continuous coefficients. Suppose a sequence $Y_{l}, l=1,2, \ldots$, of continuous 2-parameter stochastic processes on a probability space $(\Omega, \mathfrak{F}, P)$ satisfies the following conditions:

$$
\text { there exist } \gamma>0 \text { and } M>0 \text { such that }
$$

$$
\mathbf{E}\left[\left|Y_{l}(0)\right|^{\gamma}\right] \leqslant M \quad \text { for } l=1,2, \ldots,
$$

there exist $\alpha>0, \beta>0$ and $M_{T}>0$ for $T=1,2, \ldots$ such that $\mathbf{E}\left[\left|Y_{l}(z)-Y_{l}\left(z^{\prime}\right)\right|^{\alpha}\right] \leqslant M_{T} d\left(z, z^{\prime}\right)^{2+\beta}$ for $l=1,2, \ldots$ and $z, z^{\prime} \leqslant(T, T)$.

Let $P^{Y_{l}}$ be the probability distribution of $Y_{l}$ on $(W, \mathfrak{B}(W))$ for $l=1,2, \ldots$ The conditions (4.1) and (4.2) imply that the set $\left\{P^{Y_{l}}, l=1,2, \ldots\right\}$ in the space $\mathfrak{B}(W)$ of all probability measures on $(W, \mathfrak{B}(W))$ is tight. Let $\mathfrak{P}(W)$ be metrized by the Prohorov metric so that a sequence in $\mathfrak{P}(W)$ converges to an element $Q$ in $\mathfrak{P}(W)$ if and only if the sequence converges weakly to $Q$. Since $\left(W, \rho_{W}\right)$ is a complete separable metric space, the tightness of $\left\{P^{Y_{l}}, l=1,2, \ldots\right\}$ is equivalent to its relative compactness in $\mathfrak{P}(W)$. Therefore there exists $Q \in \mathfrak{P}(W)$ such that

$$
P^{Y_{(k)}} \stackrel{\text { weak }}{\rightarrow} Q \quad \text { as } k \rightarrow \infty
$$

for some subsequence $l(k), k=1,2, \ldots$ of $l=1,2, \ldots$ The statements above are extensions to 2-parameter stochastic processes of analogous statements for 1-parameter stochastic processes which are proved for instance in N. Ikeda and S. Watanabe [6]. We note that the power of the Euclidean metric on the right side of the condition (4.2) is $1+\beta, \beta>0$, for 1-parameter stochastic processes.

THEOREM 4.1. Let $\alpha, \beta \in \mathbf{M}\left(\mathbf{R}_{+}^{2} \times W\right)$ and assume that $\alpha$ and $\beta$ satisfy the continuity and order of growth conditions $(\mathrm{C} 1)$ and $(\mathrm{C} 2)$ in $\S 1$. Let $\mu$ be a probability measure on $(\mathbf{R}, \mathfrak{B}(\mathbf{R}))$ with $\int_{\mathbf{R}} x^{6} \mu(d x)<\infty$. Then the stochastic differential equation (1.1) has a solution $(X, B)$ in which $X(0)$ has $\mu$ as its probability distribution.

Proof. Let $\left(W, \mathfrak{F}_{W}, m_{W} ; \mathfrak{F}_{B, z}\right)$ be the equipped probability space with the $\left\{\widetilde{F}_{B, z}\right\}$-Brownian motion $B$ with $\partial B=0$ on it as constructed in Remark 1.2. Let $\xi$ be an $\mathfrak{F}_{B, 0}$-measurable random variable on $\left(W, \mathfrak{F}_{W}, m_{W}\right)$ whose probability distribution on $(\mathbf{R}, \mathfrak{B}(\mathbf{R}))$ is $\mu$. For $l=1,2, \ldots$, let $X_{l}$ be 2-parameter stochastic processes on ( $\left.W, \mathfrak{F}_{W}, m_{W}\right)$ satisfying (3.2), (3.3) and (3.4) with our $\xi$, where $\alpha_{l}$ and $\beta_{l}$ are defined by (3.1) with our $\alpha$ and $\beta$. The collection $\left\{X_{l}, l=1,2, \ldots\right\}$ satisfies (4.1) and (4.2) by (3.3) and Proposition 3.2 respectively. Then for the probability distributions $m_{W}^{X_{l}}$ of $X_{l}$ on $(W, \mathfrak{B}(W)), l=1,2, \ldots$, there exists a probability measure $Q$ on $(W, \mathfrak{B}(W))$ such that

$$
m_{W}^{X_{l(k)}} \stackrel{\text { weak }}{\rightarrow} Q \quad \text { as } k \rightarrow \infty
$$

for some subsequence $l(k), k=1,2, \ldots$ of $l=1,2, \ldots$.

Now a continuous 2-parameter stochastic process on the probability space $\left(W, \mathfrak{F}_{W}, m_{W}\right)$ such as our $X_{l}$ is a measurable transformation of $\left(W, \mathfrak{F}_{W}\right)$ into 
$(W, \mathfrak{A}(W))$, i.e., it is a $W$-valued random variable on $\left(W, \mathfrak{F}_{W}\right)$. Since $\left(W, \rho_{W}\right)$ is a complete separable metric space, (1) implies according to A. V. Skorokhod [12] that there exist continuous 2-parameter stochastic processes $X_{k}^{*}, k=1,2, \ldots$, and $X^{*}$ on some probability space $(\Omega, \mathfrak{F}, P)$ satisfying the conditions

$$
P^{X_{k}^{*}}=m_{W}^{X_{(k)}} \text { for } k=1,2, \ldots,
$$

where $P^{X_{k}^{*}}$ is the probability distribution of $X_{k}^{*}$ on $(W, \mathfrak{B}(W))$, and

$$
\lim _{k \rightarrow \infty} X_{k}^{*}(\cdot, \omega)=X^{*}(\cdot, \omega) \text { in }\left(W, \rho_{W}\right) \text { for a.e. } \omega \text { in }(\Omega, \mathfrak{F}, P) \text {. }
$$

(i) Let us define $M_{l(k)} \in \mathfrak{M}_{2}^{s, c}\left(\mathfrak{F}_{B, z}\right)$ for $k=1,2, \ldots$ by

$$
M_{l(k)}(z)=\int_{R_{z}} \alpha_{l(k)}\left(\zeta, X_{l(k)}\right) d B_{\zeta} .
$$

By (4) and (3.4) we have

$$
M_{l(k)}(z)=X_{l(k)}(z)-X_{l(k)}(0)-\int_{R_{z}} \beta_{l(k)}\left(\zeta, X_{l(k)}\right) d \zeta .
$$

Let us define a continuous 2-parameter stochastic process $M_{k}^{*}$ on $(\Omega, \mathfrak{F}, P)$ by

$$
M_{k}^{*}(z)=X_{k}^{*}(z)-X_{k}^{*}(0)-\int_{R_{z}} \beta_{l(k)}\left(\zeta, X_{k}^{*}\right) d \zeta .
$$

Let $\left\{\mathfrak{F}_{k, z}\right\}$ be the standard filtration on $(\Omega, \mathfrak{F}, P)$ associated with $X_{k}^{*}$ as in (1.0) for $k=1,2, \ldots$ Now $M_{k}^{*}$ is $\left\{\mathfrak{F}_{k, z}\right\}$-adapted, continuous, and square integrable, with $\partial M_{k}^{*}=0$, since the same is true of $M_{l(k)}$. To show that it is an $\left\{\mathfrak{F}_{k, z}\right\}$-strong martingale, we need only verify

$$
\mathbf{E}\left[M_{k}^{*}((\zeta, z]) \prod_{i=1}^{n} f_{i}\left(X_{k}^{*}\left(\zeta_{i}\right)\right)\right]=0
$$

whenever $\zeta<z$, each $f_{i}$ is a bounded, continuous, real valued function on $\mathbf{R}$, and $\zeta \nless \zeta_{i}$ for $i=1,2, \ldots, n$. But (7) is true, since it holds with $M_{l(k)}$ replacing $M_{k}^{*}$, and the left side of (7) depends only on the law of $X_{k}^{*}$. Similarly

$$
M_{k}^{* 2}=\int_{R_{z}} \alpha_{l(k)}\left(\zeta, X_{k}^{*}\right)^{2} d \zeta \quad \text { for } z \in \mathbf{R}_{+}^{2}
$$

is an $\left\{\mathfrak{F}_{k, z}\right\}$-martingale and therefore

$$
\left\langle M_{k}^{*}\right\rangle(z)=\int_{R_{z}} \alpha_{l(k)}\left(\zeta, X_{k}^{*}\right)^{2} d \zeta \quad \text { for } z \in \mathbf{R}_{+}^{2} .
$$

(ii) Let $\left\{\mathfrak{F}_{\infty, z}\right\}$ be the standard filtration on $(\Omega, \mathfrak{F}, P)$ associated with $X^{*}$. Define an $\left\{\mathfrak{F}_{\infty, z}\right\}$-adapted, continuous process $M^{*}$ with $\partial M^{*}=0$ on $(\Omega, \mathfrak{F}, P)$ by setting

$$
M^{*}(z)=X^{*}(z)-X^{*}(0)-\int_{R_{z}} \beta\left(\zeta, X^{*}\right) d \zeta \quad \text { for } z \in \mathbf{R}_{+}^{2} .
$$

To show that $M^{*}$ is a square integrable $\left\{\mathfrak{F}_{\infty, z}\right\}$-strong martingale, let us show first

$$
\lim _{k \rightarrow \infty} M_{k}^{*}(z)=M^{*}(z) \quad \text { for all } z \in \mathbf{R}_{+}^{2} \text { a.s. }
$$


and

$$
\sup _{k} \mathbf{E}\left[M_{k}^{*}(z)^{6}\right]<\infty \quad \text { for } z \in \mathbf{R}_{+}^{2} .
$$

Now since $[\cdot]_{l(k)}$ converges uniformly to the identity function on $[0, \infty)$ and $\beta$ is continuous, we have from (3.1) and (3)

$$
\lim _{k \rightarrow \infty} \beta_{l(k)}\left(z, X_{k}^{*}\right)=\beta\left(z, X^{*}\right) \quad \text { uniformly in } z \in R_{T, T} \text { a.s. }
$$

for every $T>0$. Therefore

$$
\lim _{k \rightarrow \infty} \int_{R_{z}} \beta_{l(k)}\left(\zeta, X_{k}^{*}\right) d \zeta=\int_{R_{z}} \beta\left(\zeta, X^{*}\right) d \zeta \quad \text { a.s. }
$$

From (5), (6), (3) and (13) we have (11). To prove (12) note that from (6)

$$
\mathbf{E}\left[M_{k}^{*}(z)^{6}\right] \leqslant 3^{6} \mathbf{E}\left[X_{k}^{*}(z)^{6}+X_{k}^{*}(0)^{6}+\left\{\int_{R_{z}} \beta_{l(k)}\left(\zeta, X_{k}^{*}\right) d \zeta\right\}^{6}\right] .
$$

For the first two terms on the right side of (14) we have by (2), (3.3) and (3.5)

$$
\mathbf{E}\left[X_{k}^{*}(z)^{6}\right]+\mathbf{E}\left[X_{k}^{*}(0)^{6}\right] \leqslant 2\left\{1+\mathbf{E}\left[\xi^{6}\right]\right\} \exp \left(2 C_{6, T} s t\right)
$$

for $z=(s, t) \leqslant(T, T)$ and $k=1,2, \ldots$ For the third term on the right side of (14) we have by applying Hölder's inequality with indices $p=6$ and $q=\frac{6}{5}$

(16)

$$
\begin{aligned}
\mathbf{E}\left[\left\{\int_{R_{z}} \beta_{l(k)}\left(\zeta, X_{k}^{*}\right) d \zeta\right\}^{6}\right] & \leqslant(s t)^{5} \int_{R_{z}} \mathbf{E}\left[\beta_{l(k)}\left(\zeta, X_{k}^{*}\right)^{6}\right] d \zeta \\
& \leqslant T^{12} K_{T} \text { for } z=(s, t) \leqslant(T, T) \text { and } k=1,2, \ldots
\end{aligned}
$$

by (12) in the proof of Proposition 3.2. By (14), (15) and (16) we have (12).

Now (12) implies the uniform integrability of $\left\{M_{k}^{*}(z)^{2}, k=1,2, \ldots\right\}$. Then by (11), $M_{k}^{*}(z)^{2}$ converges in $L^{1}(\Omega, \mathfrak{F}, P)$ to $M^{*}(z)^{2}$ and in particular

$$
\mathbf{E}\left[M^{*}(z)^{2}\right]=\lim _{k \rightarrow \infty} \mathbf{E}\left[M_{k}^{*}(z)^{2}\right]<\infty
$$

by (12), proving the square integrability of $M^{*}$.

To show that $M^{*}$ is an $\left\{\mathfrak{F}_{\infty, z}\right\}$-strong martingale, it remains to verify

$$
\mathbf{E}\left[M^{*}((\zeta, z]) \prod_{i=1}^{n} f_{i}\left(X^{*}\left(\zeta_{i}\right)\right)\right]=0,
$$

where $\zeta, z, f_{i}$ and $\zeta_{i}$ are as in (7). Now the integrand in (7) converges a.s. to that in (17) by (3) and (11). Also

$$
\begin{array}{rl}
\sup _{k} & \mathbf{E}\left[M_{k}^{*}((\zeta, z])^{6} \prod_{i=1}^{n} f_{k}\left(X_{k}^{*}\left(\zeta_{i}\right)\right)^{6}\right] \\
& \leqslant\left\{\sup _{x \in \mathbf{R}} \prod_{i=1}^{n} f_{i}(x)^{6}\right\}\left\{\sup _{k} \mathbf{E}\left[M_{k}^{*}((\zeta, z])^{6}\right]\right\}<\infty
\end{array}
$$

by (12) so that the integrands in (7) for $k=1,2, \ldots$ are uniformly integrable. Therefore by letting $k \rightarrow \infty$ in (7) we obtain (17). 
For the continuous, square integrable, $\left\{\mathfrak{F}_{\infty, z}\right\}$-strong martingale $M^{*}$, consider

$$
M^{*}(z)^{2}=\int_{R_{z}} \alpha\left(\zeta, X^{*}\right)^{2} d \zeta \quad \text { for } z \in \mathbf{R}_{+}^{2} .
$$

From (13) with $\beta$ replaced by $\alpha$, (11) and the fact that (8) is an $\left\{\mathfrak{F}_{k, z}\right\}$-martingale follows that (18) is an $\left\{\mathfrak{F}_{\infty, z}\right\}$-martingale by the same sort of argument as in showing that $M^{*}$ is an $\left\{\mathfrak{F}_{\infty, z}\right\}$-strong martingale. By (18) we then have

$$
\left\langle M^{*}\right\rangle(z)=\int_{R_{z}} \alpha\left(\zeta, X^{*}\right)^{2} d \zeta \quad \text { for } z \in \mathbf{R}_{+}^{2} .
$$

(iii) Now that (19) holds, there is an extension of the probability space $(\Omega, \mathfrak{F}, P)$ on which $X^{*}$ is based, with a Brownian motion $B^{*}$ thereon, so that

$$
M^{*}(z)=\int_{R_{z}} \alpha\left(\zeta, X^{*}\right) d B_{\zeta}^{*} \quad \text { for } z \in \mathbf{R}_{+}^{2},
$$

and hence by (10)

$$
X^{*}(z)=X^{*}(0)+\int_{R_{z}} \alpha\left(\zeta, X^{*}\right) d B_{\zeta}^{*}+\int_{R_{z}} \beta\left(\zeta, X^{*}\right) d \zeta \quad \text { for } z \in \mathbf{R}_{+}^{2},
$$

as required.

\section{REFERENCES}

1. R. Cairoli, Sur une equation differentielle stochastique, C. R. Acad. Sci. Paris Ser. A 274 (1972), 1739-1742.

2. R. Cairoli and J. B. Walsh, Stochastic integrals in the plane, Acta Math. 134 (1975), 111-183.

3. K. L. Chung and R. J. Williams, Introduction to stochastic integration. Birkhäuser, Boston, 1984.

4. C. Doléans-Dade and P. A. Meyer, Equations differentielles stochastiques, Seminaire de Probabilites XI, Lecture Notes in Math., vol. 581, Springer-Verlag, Berlin and New York, 1977.

5. X. Guyon and B. Prum, Variations-produit et formule de Ito pour les semi-martingales representables a deux parametres, Z. Wahrsch. Verw. Gebiete 56 (1981), 361-397.

6. N. Ikeda and S. Watanabe, Stochastic differential equations and diffusion processes, North-Holland, New York, 1981.

7. J. Jacod and J. Memin, Weak and strong solutions of stochastic differential equations, Stochastic Integrals, Lecture Notes in Math., vol. 851, Springer-Verlag, Berlin and New York, 1980.

8. R. S. Liptser and A. N. Shiryaev, Statistics of stochastic processes, Nauka, Moscow, 1974. (Russian)

9. P. A. Meyer, Probability and potentials, Blaisdell, Waltham, Mass., 1966.

10. J. Reid, Dissertation, University of California, Irvine, Cal., 1981.

11. Estimate on moments of the solutions to stochastic differential equations in the plane, Ann. of Probability 11 (1983), 656-668.

12. A. V. Skorokhod, Investigations on the theory of stochastic processes, Kiev University, Kiev, 1961. (Russian)

13. D. W. Stroock and S. R. S. Varadhan, Multidimensional diffusion processes, Springer-Verlag, New York, 1979.

14. C. Tudor, $A$ theorem concerning the existence of the weak solution of the stochastic equation with continuous coefficients in the plane, Rev. Roumaine Math. Pures Appl. 22 (1977), 1303-1308.

15. On the existence and the uniqueness of solutions to stochastic integral equations with two-dimensional time parameter, Rev. Roumaine Math. Pures Appl. 24 (1979), 817-827.

16. S. Watanabe, Stochastic differential equations, Sangyo-Tosho, Tokyo, 1979. (Japanese)

17. E. Wong and M. Zakai, Martingales and stochastic integrals for processes with a multi-dimensional parameter, Z. Wahrsch. Verw. Gebiete 29 (1974), 109-122.

18. J. Yeh, Existence of strong solutions for stochastic differential equations in the plane, Pacific J. Math. 97 (1981), 217-247.

DePartment of MAThematics, University of CAlifornia, IRVINe, California 92717 